\title{
Study of one-dimensional electron hopping and its effects on ESR line shape
}

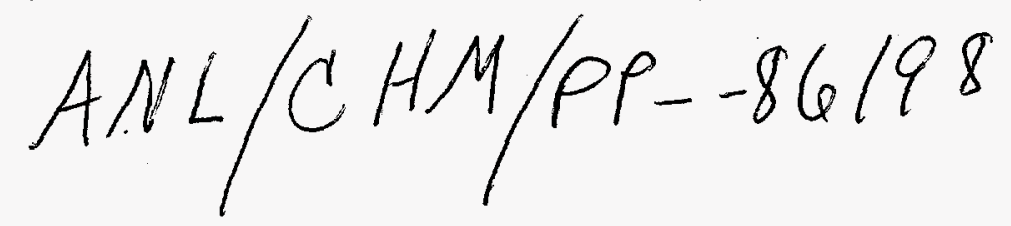

Jau Tang*, S.N. Dikshit ${ }^{+}$, and James R. Norris*+

*Argonne National Laboratory, Argonne, IL 60439

${ }^{+}$Department of Chemistry, The University of Chicago, Chicago, IL 60637

The submitted manuscript has been suthored

by controctor of the U.S. Government

by contractor of the W-31-109ENG-38.

under contract No. Wernment retains a

Aecordingly, the U. S. Government retains a

nonexclusive, royalty-lire lictnse to publish

or reproduce the published form of this

U.S. Government purposes. 


\section{DISCLAMMER}

Portions of this document may be illegible in electronic image products. Images are produced from the best available original document. 


\title{
Abstract
}

Random hopping processes between discrete sites along a finite open chain or around a closed finite loop are examined. Closed form formulae are prescribed for the dependence of the ESR (electron spin resonance) line shape on the chain length and hopping rate. Significant differences between the closed loop and open chain are demonstrated. Deviation at short time from the results of diffusion in a continuum is presented.

\section{DISCLAIMER}

\begin{abstract}
This report was prepared as an account of work sponsored by an agency of the United States Government. Neither the United States Government nor any agency thereof, nor any of their employees, makes any warranty, express or implied, or assumes any legal liability or responsibility for the accuracy, completeness, or usefulness of any information, apparatus, product, or process disclosed, or represents that its use would not infringe privately owned rights. Reference herein to any specific commercial product, process, or service by trade name, trademark, manufacturer, or otherwise does not necessarily constitute or imply its endorsement, recommendation, or favoring by the United States Government or any agency thereof. The views and opinions of authors expressed herein do not necessarily state or reflect those of the United States Government or any agency thereof.
\end{abstract}


Since the earlier treatment by Einstein, the theory of Brownian motion has been generalized and extended by Smoluchowski. Additional important contributions have been made by many others. ${ }^{1-3}$ Random walk processes for an unpaired electron can be studied by EPR (electron spin resonance) using line shape analysis. For example, magnetic resonance has been used to investigate exchange narrowing in $\left(\mathrm{CH}_{3}\right) \mathrm{NMnCl}_{3}$ (TMMC) which exhibits special long-time spincorrelations as evidence for the one-dimensional exchange for such an antiferromagnetic system. ${ }^{4}$ More recently, the ESR line shape study of transpolyacetylene has also provided evidence for soliton diffusion along a onedimensional chain. ${ }^{5-9}$ A reduction of the second moment of the ESR spectrum for the primary donor in photosynthetic reaction centers provided the crucial evidence for the dimeric nature of the special pair. ${ }^{10}$ Recent ESR measurements on antenna in the light-harvesting complexes of bacterial photosynthesis may indicate electron hopping among many equivalent sites. ${ }^{11-13}$ In this letter, we shall examine two cases of one-dimensional random jump processes, one along a finite closed loop and the other along an open chain. Additionally we derive the effect of such diffusion on the ESR line shape. The dynamics of random hopping along a $\mathrm{N}$-site chain will be shown to be topologically equivalent to a $2 \mathrm{~N}$-site closed loop formed by joining the $\mathrm{N}$-site chain with its image $\mathrm{N}$-site chain. Analytical expressions will be derived for the dependence of the ESR line shape on the chain length and hopping rate, showing significant differences between a short length closed loop and open chain. For an infinitive loop or chain, it will be shown that the long time behaviors are identical for both discrete hopping model and diffusion equation in a continuum. However, deviation exists at the short time. In many analyses of ESR lineshapes ${ }^{5-9}$ or femtosecond excitation-state relaxation ${ }^{14-16}$ in one-dimensional systems the continuum model is often used which is valid only for an infinite chain 
and is accurate only at long time. This work considering discrete hopping along a finite chain or closed loop offers a more accurate account of short time behaviors.

First of all, we shall consider nearest-neighbor hopping around an $\mathrm{N}$-site closed loop. The rate equation for population at the $n$-th site $f_{n}(\tau)$ is given by

$$
\frac{d}{d \tau} \hat{f}_{n}=-2 W f_{n}+W f_{n-1}+W f_{n+1}, \quad(n=0,1,2, \ldots, N-1)
$$

where $W$ is the hopping rate. Using the cyclic condition that $f_{n+N}=f_{n}$ and assuming a particle initially located at the $m$-th site, the probability of finding the particle at the $n$-th site $f_{n}(\tau)$ can be obtained in a closed form

$$
f_{n}(\tau)=\frac{1}{N} \sum_{k=0}^{N-1} e^{-4 W \tau \sin ^{2}\left(\frac{\pi k}{N}\right)} \cos \left[\frac{2 \pi k}{N}(n-m)\right] .
$$

The auto-correlation function $\mathrm{g}_{\mathrm{N}}(\tau)$, the probability of finding a particle at the $\mathrm{m}$-th site at time $\tau$, is given by

$$
g_{N}(\tau)=\frac{1}{N} \sum_{k=0}^{N-1} e^{-4 \omega \tau \sin ^{2}\left(\frac{\pi k}{N}\right)}
$$

As illustrated in fig. $1, \mathrm{~g}_{\mathrm{N}}(\tau)$ generally displays an initial sharp decay and approaches $1 / \mathrm{N}$ at a later time with a long tail especially if $\mathrm{N}$ is sufficiently large. For an infinite chain, one can show from eq. that (3) $\mathrm{g}_{\infty}(\tau)=\mathrm{I}_{0}(2 \mathrm{~W} \tau) \exp (-2 \mathrm{~W} \tau)$, where $I_{0}$ is the modified Bessel function of the second kind. For a large $W \tau, g_{\infty}(\tau)$ $\simeq 1 /(4 \pi \mathrm{W} \tau)^{1 / 2}$. This long time limit agrees with the well-known result of the onedimensional diffusion equation in a continuum. However, the often used form ${ }^{7,8}$ 
of $g_{\infty}(\tau)$ by $1 /(1+4 \pi \mathrm{W} \tau)^{1 / 2}$ at a shorter time deviates from the $\mathrm{I}_{0}(2 \mathrm{~W} \tau) \exp (-2 \mathrm{~W} \tau)$ derived here.

As the unpaired electron hops around the closed loop, it experiences a fluctuating hyperfine field resulting from a different nuclear spin configuration at each site. According to Kubo ${ }^{17,18}$, the corresponding ESR spectral function for such a system can be calculated from the Fourier transform of the following response function in time $S_{N}(t)$, and

$$
\begin{aligned}
S_{N}(t) & =\exp \left[-\sum_{\alpha} \frac{1}{3} I_{\alpha}\left(I_{\alpha}+1\right) a_{\alpha}^{2} \int_{0}^{t}(t-\tau) g_{N}(\tau) d \tau\right] \\
= & \exp \left\{-\frac{\Delta \omega^{2}}{N} \sum_{k=0}^{N-1} \frac{1}{15 W^{2} \sin ^{4}\left(\frac{\pi k}{N}\right)}\left[e^{-4 W t \sin ^{2} \frac{\pi k}{N}}+4 W t \sin ^{2} \frac{\pi k}{N}-1\right]\right\}
\end{aligned}
$$

where $\Delta \omega^{2}$, defined as $\left\langle\sum_{\alpha} I_{\alpha}\left(I_{\alpha}+1\right) a_{\alpha}{ }^{2} / 3\right\rangle$, is the mean squares of the nuclear hyperfine couplings. In fig. 2 , the response function $S_{24}(t)$ is illustrated for various hopping rates $\mathrm{W}$. The corresponding spectral functions, Fourier transform of $\mathrm{S}_{24}(\mathrm{t})$, for both the slow and fast hopping limits are Gaussian with a second moment given by $\Delta \omega^{2}$ and $\Delta \omega^{2} / 24$, respectively. For a general $N$, it can be shown from eq. (4) that $S_{N}(t) \approx \exp \left(-\Delta \omega^{2} t^{2} / 2\right)$ if $W t * 1$ and $\exp \left(-\Delta \omega^{2} t^{2} / 2 N\right)$ if $W t » 1$. Thus, the second moment of the corresponding ESR spectrum is reduced by a factor of $N$ in the fast hopping limit for a $\mathrm{N}$-site closed loop. For an intermediate rate $\mathrm{W}, \mathrm{S}_{\mathrm{N}}(\mathrm{t})$ or its spectral function is generally non-Gaussian. In fig. 3, the semi-logarithmic plot of $S_{6}(t)$ versus $t^{2}$ is shown. The linear curves for both very slow or very fast hopping rates indicate a Gaussian ESR line shape; however, for intermediate rate the non-linear relationship indicates a non-Gaussian spectral line shape. For an 
infinite chain $S_{\infty}(t) \simeq \exp \left(-2 / 3 \Delta \omega^{2} t^{3 / 2} /(\pi W)^{1 / 2}\right)$ for $W t$ " 1 , and such a $t^{3 / 2}$-dependence in the exponent is the characteristics for one-dimensional diffusion processes.

Now, we shall consider the other case of random hopping processes along an open $\mathrm{N}$-site chain. The rate equation is given by

$$
\begin{aligned}
& \frac{d}{d \tau} f_{n}=-2 W f_{n}+W f_{n-1}+W f_{n+1}, \quad(n=1,2, \ldots, N-2) \\
& \frac{d}{d \tau} f_{0}=-W f_{0}+W f_{1}, \\
& \frac{d}{d \tau} f_{N-1}=-W f_{N-1}+W f_{N-2},
\end{aligned}
$$

The boundary effects at $\mathrm{n}=0$ and $\mathrm{N}-1$ can be circumvented using the following method. By adding $\mathrm{N}$ fictitious sites with $\mathrm{f}_{2 \mathrm{~N}-\mathrm{n}}=\mathrm{f}_{\mathrm{n}-1}$ and connecting both ends of this image chain, eq. (5) can be transformed into

$$
\frac{d}{d \tau} f_{n}=-2 W f_{n}+W f_{n-1}+W f_{n+1}, \quad \text { in }=0,1,2, \ldots, 2 N-1 \text { ) }
$$

where $f_{2 N+n}=f_{n}$. The above equation for random hopping around a $2 N$-site closed loop, indicates an interesting topological equivalence between the open chain and the closed loop. Thus, the solution for eq. (5), or equivalently for eq. (6), can also be obtained in the following closed form 


$$
\begin{aligned}
f_{n}(\tau) & =\frac{1}{N N} \sum_{m=0}^{N-1} f_{n=0}(0)+ \\
& \frac{2}{N} \sum_{m=0}^{N-1} f_{m}(0) \sum_{k=1}^{N-1} \cos \left[\frac{\pi k}{N}\left(m+\frac{1}{2}\right)\right] \cos \left[\frac{\pi k}{N}\left(n+\frac{1}{2}\right)\right] e^{-2 k \pi\left[1-\cos \left(\frac{\pi k}{N}\right)\right]} .
\end{aligned}
$$

Assuming an initial population at the $n$-th site, the auto-correlation function $\mathrm{g}_{\mathrm{N} . \mathrm{n}}(\tau)$ can be expressed by

$$
g_{N, n}(\tau)=\frac{1}{N}+\frac{2}{N} \sum_{k=1}^{N-1} \cos ^{2}\left[\frac{\pi k}{N}\left(n+\frac{1}{2}\right)\right] e^{-4 w \tau \sin ^{2}\left(\frac{\pi k}{2 N}\right)}
$$

Unlike the case for a closed loop, $\mathrm{g}_{\mathrm{N} . \mathrm{n}}(\tau)$ in the above equation depends also on the initial site location $\mathrm{n}$ in addition to the dependence on chain-size $\mathrm{N}$. The response function in time $S_{N}(t)$ is given by

$$
\begin{aligned}
& S_{N}(t)=\frac{1}{N} \sum_{n=0}^{N-1} S_{N, n}(t), \\
& S_{N, n}(t)=\exp \left\{-\frac{\Delta \omega^{2}}{N}\left[\frac{t^{2}}{2}+\sum_{k=1}^{N-1}\left(1+\cos \left(\frac{2 \pi k\left(n+\frac{1}{2}\right)}{N}\right)\right)\right.\right. \\
& \left.\left.\left.\cdot \frac{1}{16 W^{2} \sin ^{4}\left(\frac{\pi k}{2 N}\right)} i e^{-4 W t \sin ^{2} \frac{\pi k}{2 N}}+4 W t \sin ^{2} \frac{\pi k}{2 N}-1\right)\right]\right\} .
\end{aligned}
$$

Because $S_{N . n}(t)$ depends on the initial site $n$, to calculate an overall $S_{N}(t)$ an esemble average has to be taken for all possible sites. The individual response function $S_{6, n}(t)$ for a six-site open chain is illustrated in fig. 4. The narrowing of $S_{6, n}(t)$ for $n=0$, or 5 as compared to $\mathrm{n}=2$ or 3 indicates a larger spectral moment for its 
corresponding ESR line shape if the initial site is closer to the end of a chain.

In conclusion, both cases of one-dimensional random hopping processes around a $\mathrm{N}$-site closed loop and open chain are considered. Topological equivalence between these two cases is demonstrated by connecting an open chain with its fictitious image chain at both ends. The boundary problems in eq. (5) can then be circumvented. The distribution function $\mathrm{f}_{\mathrm{n}}(\tau)$, the auto-correlation function $\mathrm{g}_{\mathrm{N}, \mathrm{n}}(\tau)$ and the response function $S_{N}(t)$ are derived in a closed form for both systems. This work also provides a general description of the ESR line shape from the slow to fast hopping limits. It is shown that fast hopping causes a reduction of the second moment by $1 / \mathrm{N}$. For an intermediate hopping rate W, the ESR line shape deviates significantly from a Gaussian. For an infinitive loop or chain, the long time behaviors are identical for both discrete hopping model and diffusion equation in a continuum. However, deviation exists at the short time. Using the continuum model to interpret the ESR measurements of soliton diffusion in transpolyacetylene ${ }^{7,8}$ or exchange narrowing in $\mathrm{TMMC}^{4}$ may lead to an underestimate of the rate constant $\mathrm{W}$ for hopping processes which involve discrete sites. Further ESR experiments on antenna complexes of bacterial photosynthetic protein can provide direct test and demonstration of one-dimensional random walk processes around a finite closed loop or open chain of discrete sites. Measurements of both protonated and deuterated systems at various temperature can provide information about the number of the sites in the closed loop or open chain and the hopping rate.

\section{Acknowledgment}

This work was supported by the U.S. Department of Energy, Office of 
Basic Energy Sciences, Division of Chemical Sciences, under Contract W-31109-Eng-38. 
Figure caption

Fig. 1. The time dependence of the auto-correlation $\mathrm{g}_{\mathrm{N}}(\tau)$ for a $\mathrm{N}$-site closed loop with $\mathrm{N}=2,3,4,5,6$, and 24 .

Fig. 3. The response function $S_{24}(t)$ of a twenty-four-site closed loop, and $\Delta \omega / \mathrm{W}=$ $10^{2}, 10^{1}, 1,10^{-1}$, and $10^{-2}$ for curves from a to e, respectively.

Fig. 3. The dependence of $-\ln \left[\mathrm{S}_{6}(\mathrm{t})\right]$ for a six-site closed loop on $\Delta \omega^{2} \mathrm{t}^{2}$, and $\Delta \omega / \mathrm{W}$ $=10^{2}, 10^{1}, 1,10^{-1}$, and $10^{-2}$ for curves from a to e, respectively. The relative slopes of both slow and fast extremes shows a reduction in the spectral second moment by $1 / 6$ due to fast hopping.

Fig. 4. The response function $S_{6, n}(t)$ for a six-site open chain, $\Delta \omega / \mathrm{W}=0.1$. Curve a represents $\mathrm{n}=0$ and 5 , curve $\mathrm{b}$ for $\mathrm{n}=1$ and 4 , and curve $\mathrm{c}$ for 2 and 3 , respectively. $S_{6, n}(t)$ decays in time faster if the initial site is closer to the end of a chain. 
References

[1] G. E. Uhlenbeck and L. S. Ornstein, Phys. Rev. 36, 823 (1930).

[2] M. C. Wang and G. E. Uhlenbeck, Rev. Mod. Phys. 17, 323 (1945).

[3] N. Wax, ed., "Selected Papers on Noise and Stochastic Processes" (Dover Publications, New York, 1954).

[4] R. E. Dietz, F. R. Merritt, R. Dingle, D. Hone, B. G. Silbernagel, and P. M. Richards, Phys. Rev. Lett. 26, 1186 (1971).

[5] Nechtschein, F. Devreux, R. L. Greene, T. C. Clarke, and G. B. Street, Phys. Rev. Lett. 44, 356 (1980).

[6] R. Weinberger, E. Ehrenfreund, A. Pron, A. J. Heeger, and A. G. MacDiarmid, J. Chem. Phys. 72, 4749 (1980).

[7] N. S. Shiren, Y. Tomkiewicz, T. G. Kazyaka, A. R. Taranko, H. Thomann, L. Dalton, T. C. Clarke, Solid State Comm. 44, 1157 (1982).

[8] J. Tang, C. P. Lin, M. K. Bowman, J. R. Norris, J. Isoya, and H. Shirakawa, Phys. Rev. B28, 2845 (1983).

[9] P. K. Kahol and M. Mehring, J. Phys. C: Solid State Phys. 19, 1045 (1986). 
[10] J. R. Norris, R. A. Uphaus, H. L. Crespi and J. J. Katz, Proc. Nat. Acad. Sci. USA 68, 625 (1971).

[11] I. Gomez, C. Sieiro, J. M. Ramirez, S. Gomez-Amores and F. E. del Campo, FEBS Lett. 144, 117 (1982).

[12] G. Gingras and R. Picorel, Proc. Natl. Acad. Sci. USA 87, 3405 (1990).

[13] S.N. Dikshit, D. M. Tiede, J. Tang and J. R. Norris (to be published).

[14] C. V. Shank, R. Yen, and R. L. Fork, Phys. Rev. Lett. 49, 1660 (1982).

[15] Z. Vardeny, J. Strait, D. Moses, T.-C. Chung, and A. J. Heeger, Phys. Rev. Lett. 49, 1657 (1982).

[16] C. V. Shank, R. Yen, J. Orenstein, and G. L. Baker, Phys. Rev. B 28, 6095 (1983).

[17] R. Kubo, Fluctuation, relaxation, and resonance in magnetic systems, ed. D. Ter Haar (Oliver and Boyd, Edinburge, 1962).

[18] R. Kubo, J. Math. Phys. 4, 174 (1963). 


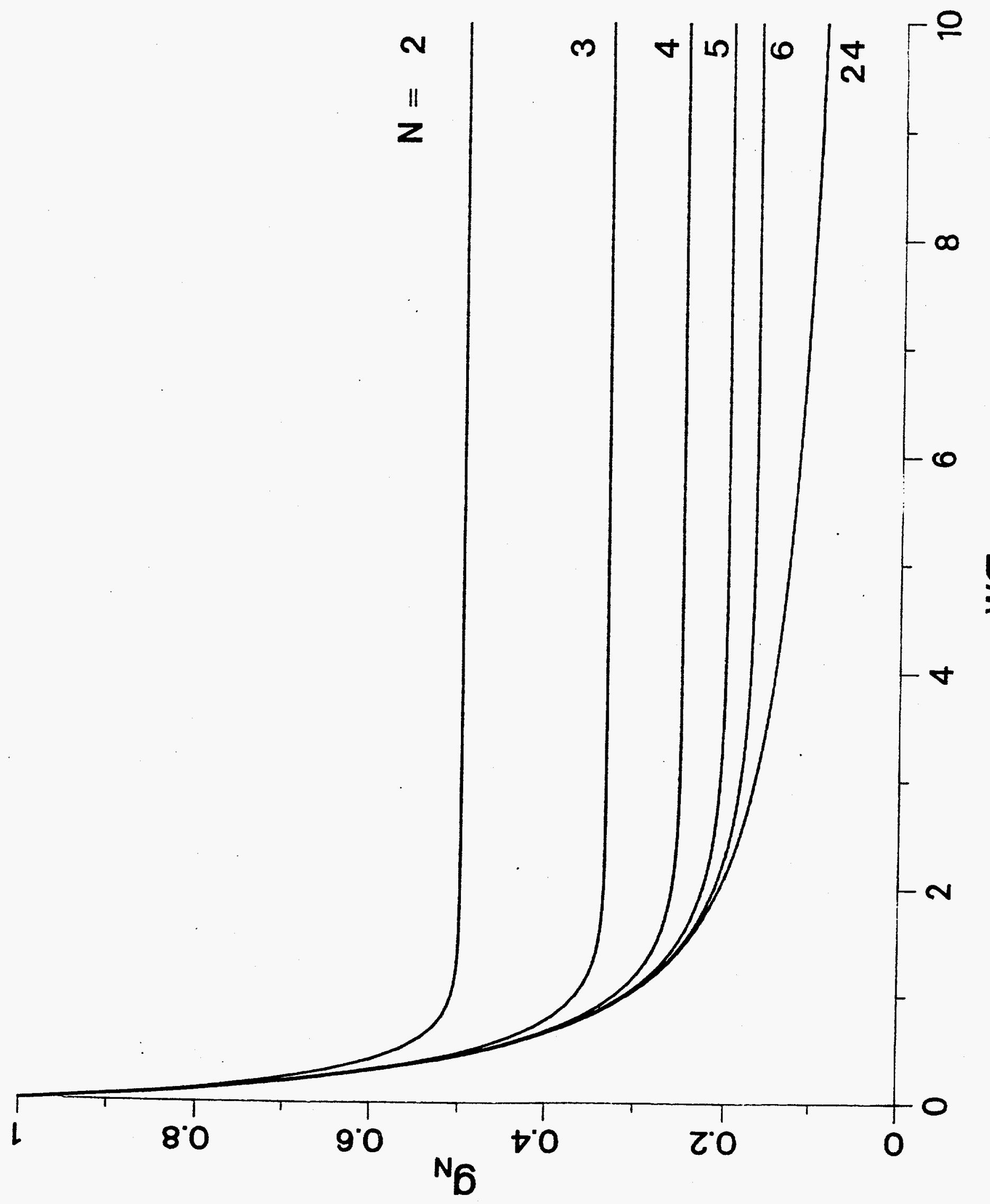




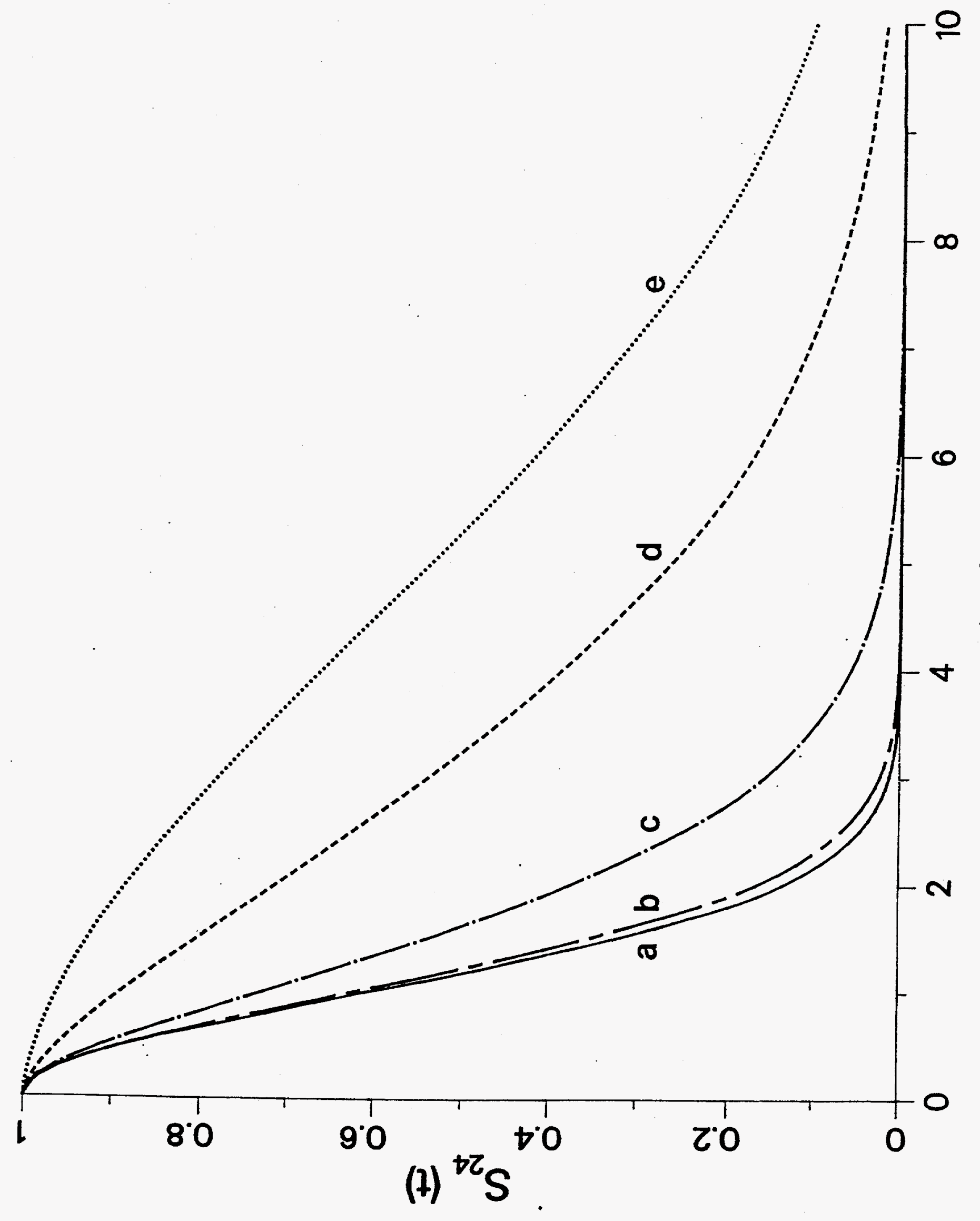




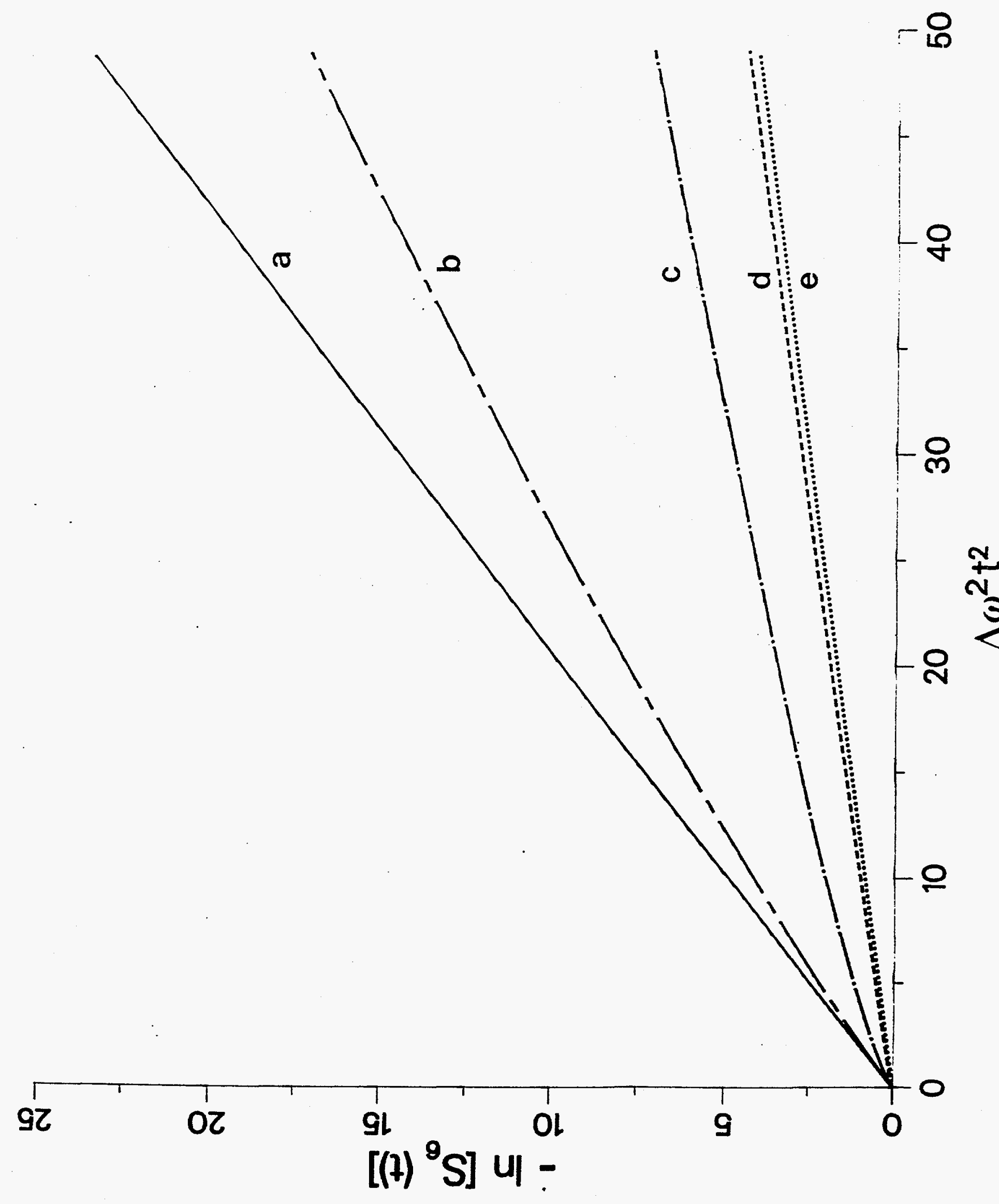




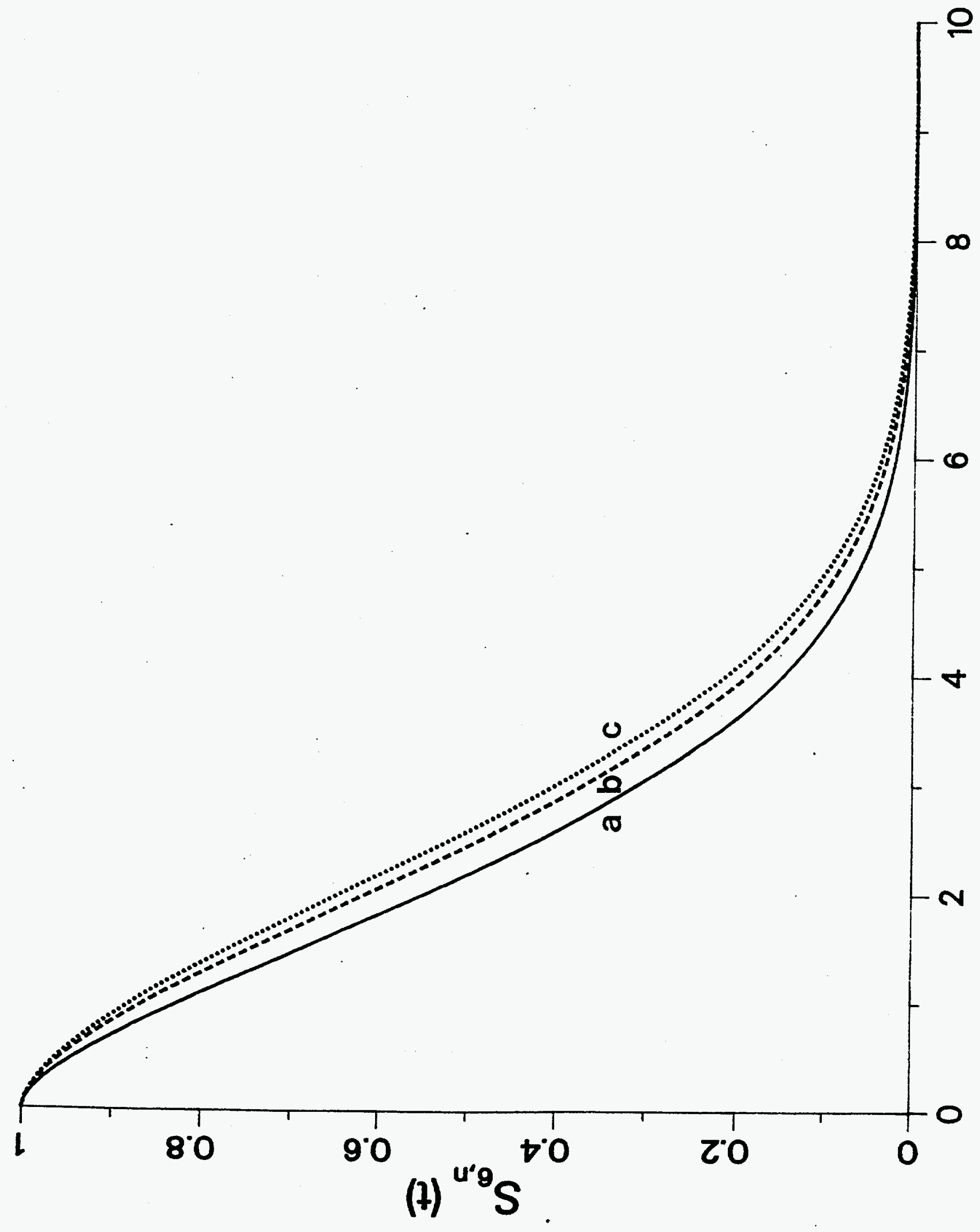

